\title{
Testing effects on hardness of a Zr-based metallic glass under nanoindentation
}

\author{
M.C. Li ${ }^{\text {a }}$, M.Q. Jiang b,c, F. Jiang ${ }^{\text {a,* }}$, L. He ${ }^{\text {a }}$, J. Sun ${ }^{\mathrm{a}}$ \\ a State Key Laboratory for Mechanical Behavior of Materials, Xi'an Jiaotong University, Xi'an 710049, China \\ b State Key Laboratory of Nonlinear Mechanics, Institute of Mechanics, Chinese Academy of Sciences, Beijing 100190, China \\ c School of Engineering Science, University of Chinese Academy of Sciences, Beijing 100049, China
}

\section{A R T I C L E I N F O}

\section{Article history:}

Received 11 April 2017

Received in revised form 24 May 2017

Accepted 30 May 2017

Available online 7 June 2017

Keywords:

Metallic glass

Nanoindentation

Strain rate

Indentation size effect

\begin{abstract}
A B S T R A C T
A series of nanoindentation tests were performed on a Zr-based bulk metallic glass at different peak-loads and strain rates. It is observed that the strain rate sensitivity index gradually decreases from positive to almost zero with increasing peak-load. Furthermore, at a fixed strain rate, the indentation hardness firstly shows a dramatic reduction and then becomes stable with increasing peak-load. These observations can be well understood in the framework of the activation and the accumulation of free volume via shear transformation zones. The current work may provide a practical way to tailor the hardness of the metallic glass.
\end{abstract}

(c) 2017 Acta Materialia Inc. Published by Elsevier Ltd. All rights reserved.
Metallic glasses have been long regarded as potential structural materials due to their ultra-high strength and hardness [1,2]. Therefore, a number of researchers have paid much attention to the factors that will influence the hardness of metallic glass [3-5]. For instance, it is demonstrated that the increase of loading rate corresponded to a decrease of hardness and the underlying mechanism was attributed to the free volume relaxation induced hardening that was more dramatic under lower strain rates [6]. However, the free volume dynamics includes not only the relaxation induced annihilation, but also stress driven creation [7-9]. It is believed that the free volume creation could lead to a softening effect on the hardness of metallic glasses [10,11]. Therefore, both hardening and softening effects of strain rates on the hardness is possible. For example, Xue et al. [12] reported a positive strain rate dependence of the hardness and ascribed this correlation to the stronger strain rate softening effect under lower strain rates. There are also analogous inconsistent results, but the underlying mechanism has been rarely shed light on [13-17]. In addition, Bhattacharyya et al. [6] demonstrated a lower strain rate sensitivity of the hardness under a higher peak-load, but the results of Ma et al. [17,18] showed a much more positive rate sensitivity when the peak-load increased by 1 order of magnitude. Moreover, Jang et al. [19] reported a continuous reduction of

\footnotetext{
* Corresponding author.

E-mail address: jiangfeng@mail.xjtu.edu.cn (F. Jiang).
}

hardness with increasing peak-load or indentation size, i.e., an indentation size effect (ISE). On the contrary, Wang et al. [20] recently demonstrated that the ISE would disappear when the indentation size is larger than a critical value.

Evidently, the dependence of hardness on the testing conditions (strain rate and peak-load) under nanoindentation is still under debate. Further exploration is required to gain more insights into these issues. In the current work, a series of nanoindentation tests were performed on a Zr-based metallic glass under different peak-loads and strain rates. A positive dependence of hardness on the strain rate is found. Meanwhile, both the strain rate sensitivity index and the indentation hardness firstly decrease and then become stable with the increasing peak-load.

Nanoindentation studies were performed on a fully amorphous metallic glass specimen with the nominal composition of $\mathrm{Zr}_{52.5} \mathrm{Cu}_{17.9} \mathrm{Ni}_{14.6} \mathrm{Al}_{10} \mathrm{Ti}_{5}$. Prior to the indentation tests, the surfaces of the specimen were ground and polished to a mirror finish. All of the nanoindentations were carried out with a load-controlled mode in a Nanovea system equipped with a standard Berkovich diamond tip indenter. The peak-loads, $P_{\max }$, were fixed at $30 \mathrm{mN}, 60 \mathrm{mN}, 100 \mathrm{mN}$, $200 \mathrm{mN}$ and $300 \mathrm{mN}$, respectively. For each peak load, four different loading rates, $d P / d t$, were carried out, i.e., $P_{\max } / 300, P_{\max } / 150, P_{\max } / 30$, and $P_{\max } / 15 \mathrm{mN} / \mathrm{s}$, respectively. In order to evaluate the strain rate sensitivity, all of the loading rates were converted into the equivalent strain rate as: $\dot{\varepsilon}=\left(1 / 2 P_{\max }\right)(d P / d t)$. [13]. Then we obtain four equivalent strain rates: $1 / 600 \mathrm{~s}^{-1}, 1 / 300 \mathrm{~s}^{-1}, 1 / 60 \mathrm{~s}-1$ and $1 / 30 \mathrm{~s}^{-1}$, respectively, for 

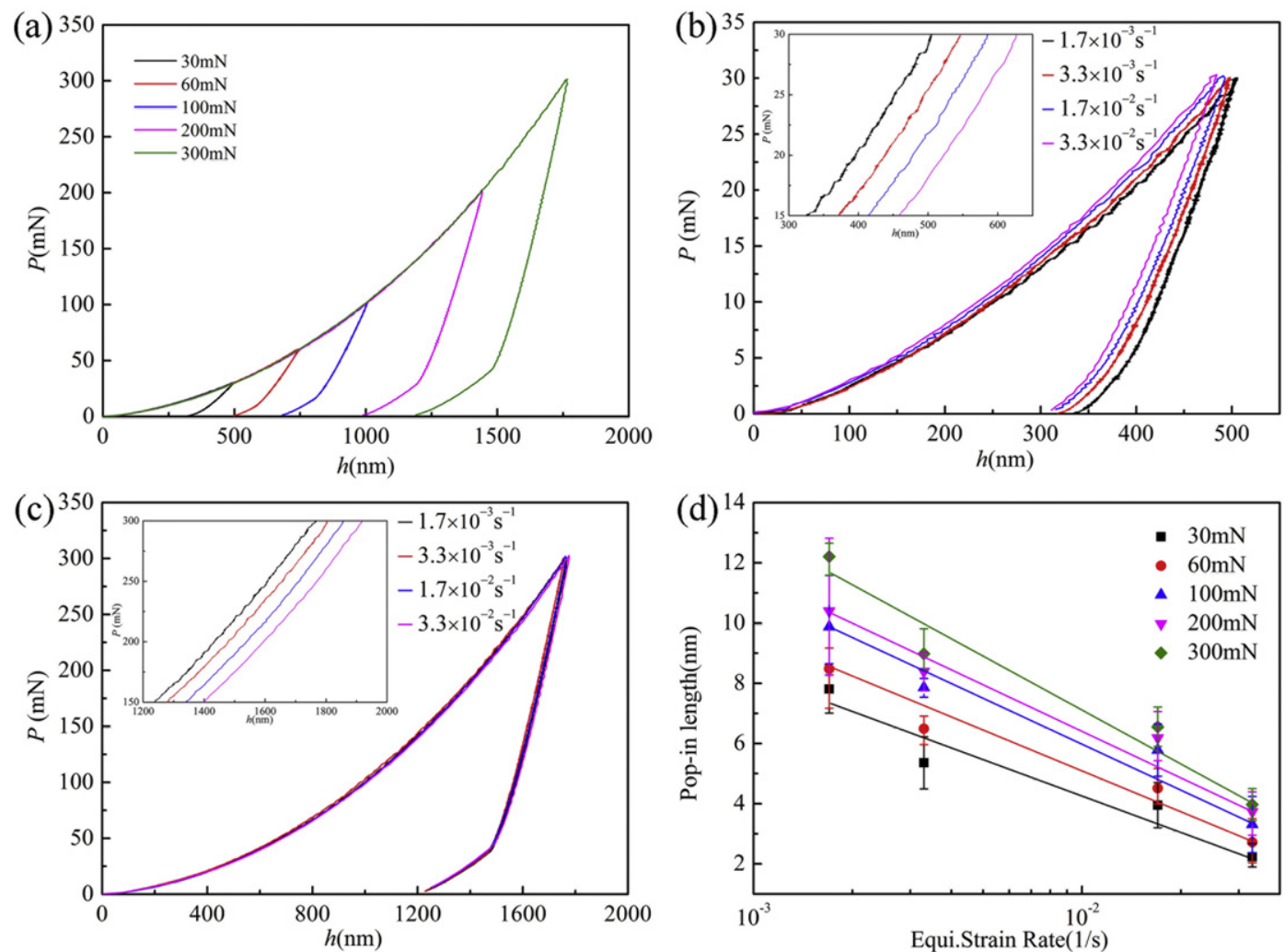

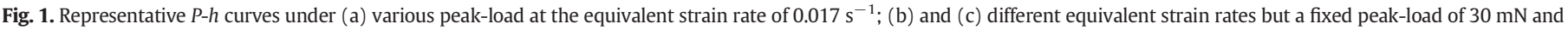
$300 \mathrm{mN}$, respectively. (d) Variation of pop-ins with peak-load and strain rate.

each peak load. To rule out the overlap of the plastic regions between two adjacent indentations, each of the indentations was separated by lateral distance of $20 \mu \mathrm{m}$. For the sake of ensuring the reliability of the experimental data, the arrays with $4 \times 4$ indentations were performed on the areas far from the bulk boundary under each case by the way of nesting the mutual matrixes. After tests, the surface morphologies of the indentations were observed by scanning electron microscopy (SEM; SU6600).

Fig. 1a shows the representative nanoindentation loaddisplacement $(P-h)$ curves under different peak-loads but a fixed equivalent strain rate of $0.017 \mathrm{~s}^{-1}$. All curves display the parabolic shape at the loading stage. During the unloading process, the $P$ - $h$ curves show a similar linear-unloading behavior during the upper portion and then followed by a nonlinear-unloading response stemming from the residually plastic deformation. This indicates that the Oliver and Pharr method [21], which was derived from purely elastic solution, may not be reasonable for the hardness calculation in the present case. Moreover, it is addressed that the Meyer's hardness method can correct the minor error due to the pile-up effect $[6,19]$. Therefore, Meyer's hardness that is defined as $H=P_{\max } / A_{t}$ is adopted. Here, $A_{t}$ is the true contact area that is calculated as $A_{t}=3 \sqrt{3} a^{2} / 4$, where $a$ is the averaged length measured from the center of the triangular impression to the corner with the aid of SEM [19] (see Fig. S1 in Supplementary material). Fig. 1b and c demonstrate the $P$ - $h$ curves of $30 \mathrm{mN}$ and $300 \mathrm{mN}$ under different strain rates. Obviously, the curvature of the $P$ - $h$ curves under $30 \mathrm{mN}$ during loading segment has a slighter increase with increasing strain rate, which means a positive strain rate dependence of hardness. However, the difference in curvature is nearly invisible under $300 \mathrm{mN}$, implying the absence of strain rate effect. The inset in Fig. 1b and c shows the local magnification of loading segment. There are apparent displacement bursts, i.e., pop-ins, during the loading segment especially at lower strain rates. Fig. 1d presents the decrease of the average length of pop-in with either the increasing strain rate or the decreasing peakload. This considerable variance may be attributed to the heterogeneous flow of the material under the indenter [15,22].

Fig. 2a presents the variation of hardness with peak-load and strain rate in a double-logarithmic coordinates. Apparently, the indentation hardness has a positive dependence on strain rate. Moreover, the strain rate sensitivity index $(m)$ of hardness, i.e., the slop of the dotted line, firstly has a dramatic reduction and then becomes close to zero with increasing peak-load. This indicates that the hardness will be independent on strain rate when the peak-load increases to a suitable high level. Fig. 2a also demonstrates a lower hardness under a higher peak-load, i.e., an indentation size effect (ISE). The dependence of hardness on peak-load and the corresponding indentation depth at the strain rate of $0.017 \mathrm{~s}^{-1}$ is diagramed in Fig. $2 \mathrm{~b}$ (the ISE under other strain rates are similar and hence not presented). Evidently, the hardness firstly has a dramatic reduction and then becomes nearly stable with the increasing peak-load (or indentation depth). Furthermore, an approximately parabolic correlation can be found between hardness and indentation depth, which is presented as the solid line (the dotted line in the $\mathrm{H}-\mathrm{P}$ data is just guide for the eye). The hardness corresponds to the vertex of the parabola is $4.94 \mathrm{GPa}$, which is very close to that acquired under $300 \mathrm{mN}$. This means that the indentation hardness will level off rather than continuously reduce when the peak-load is larger than a critical value.

To have a further insight into the dependence of hardness on the strain rate and peak-load, micro-indentation tests with a peak-load of $20 \mathrm{~N}$ under four strain rates were carried out on the same specimen. As presented in Fig. 3, the value of $m$ is about $2.6 \times 10^{-4}$, which confirms the absence of the strain rate effect on the hardness. Moreover, the hardness is about $4.95 \mathrm{GPa}$, which is very close to that observed under 

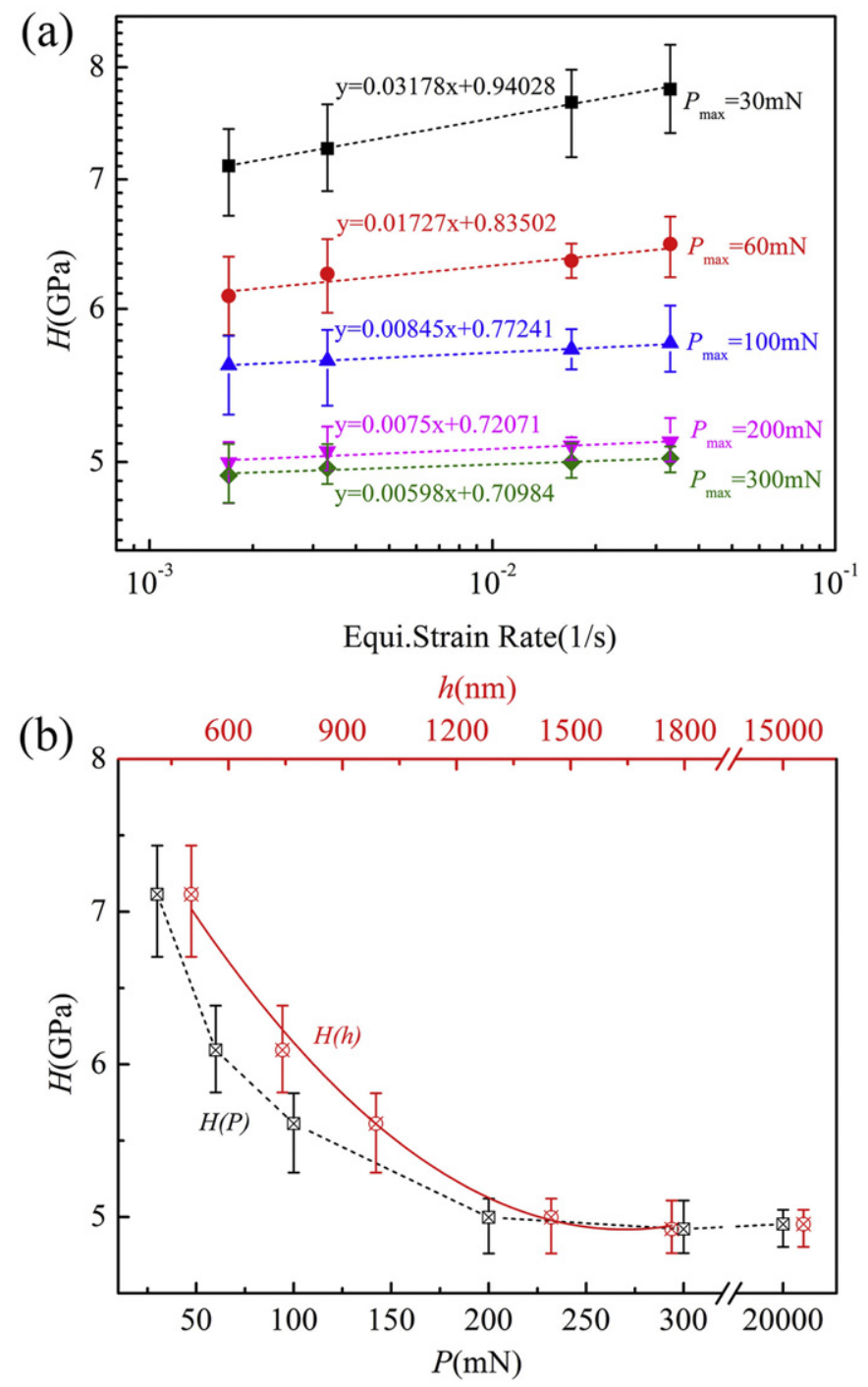

Fig. 2. Variation of hardness with (a) different equivalent strain rate under a series of peakloads; (b) peak-load and the corresponding indentation depth.

$300 \mathrm{mN}$. Therefore, it is rational to conclude that both the strain rate sensitivity and the ISE of the metallic glass will tend to be insignificant when the peak-load increases to a suitable high level.

It is accepted that metallic glasses can be regarded as a material consisting of elastic matrix and liquid-like regions with high density of free volume $[23,24]$. Upon loading, the liquid-like regions will act as the potential sites of shear transformation zones (STZs), which are the unit carriers of plasticity in metallic glasses $[25,26]$. As the external stress keeps increasing, more and more STZs will be activated, and meanwhile the associated atomic rearrangements in the STZs can relax the local stress and reduce the local energy concentration. In order to counterbalance this drop in local stress, atoms in the surrounding region will be absorbed into the STZs. When the external stress increases to a critical value, the activated STZs will expand outwards and combine with each other. It leads to the formation of one mature shear band. Then the elastic backbone will be penetrated and give rise to irreversible macroscopic yield of the metallic glass [20,27,28]. The critical stress corresponds to the yield strength or the hardness. Under higher strain rates, a higher stress can be reached within the same loading time, thus triggering faster STZs and consequently plasticity develops faster. Due to shear-induced dilatation effect [29], more free volume can be generated, which gives rise to a more pronounced strain softening (stress drop). However, at higher strain rates, the activation of

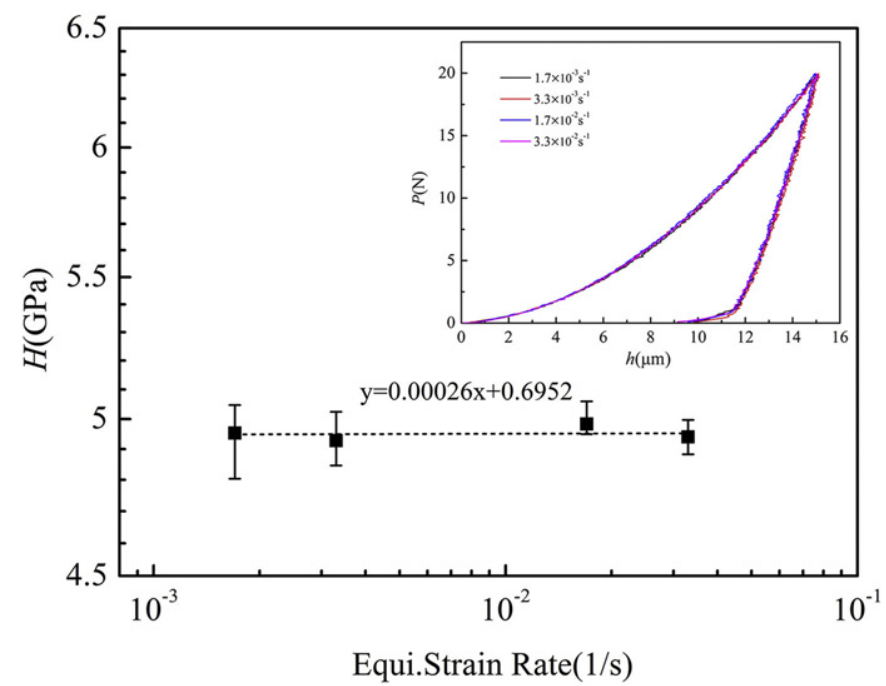

Fig. 3. The variation of micro-indentation hardness on equivalent strain rate under $20 \mathrm{~N}$. The insert presents the representative $P$ - $h$ responses under four strain rates.

STZs and free volume creation are delayed due to short time. This results in a higher yield stress before strain softening (stress drop), and thus a more remarkable stress overshoot is observed at higher strain rates [8, $20,30,31]$. Therefore, with increasing strain rate, the yield stress to activate STZs increases, although strain softening becomes more pronounced. This explains the positive dependence of hardness on the strain rate observed in Fig. 2a. After yielding, the higher stress overshoot under higher strain rate corresponds to larger amount of activated STZs, which in turn leads to a higher net creation of free volume. With increasing strain rate, the STZ and the free volume could prompt each other with a positive feedback [8]. The larger amount of activated STZs will contribute to multiple shear bands. During nanoindentation, the total plastic displacement is $h_{p}=N_{P} \Delta h$, where $\Delta h$ and $N_{P}$ are the average pop-in displacement of all indentation depths and the shear band density, respectively [22]. For a fixed peak-load but different strain rates, the total plastic displacement is approximately equal, then the average strain carried by each shear band under a higher strain rate is smaller than that under a lower one. This is in line with the smoother $P$ - $h$ curves (Fig. $1 \mathrm{~b}$ and $\mathrm{c}$ ) and the smaller pop-in length (Fig. 1d) at higher strain rates.

The dependence of $m$ on peak-load can also be understood in the framework of free volume dynamics via STZs. For a low peak-load indentation, the local plastic zone underneath the indenter is relatively small. Then the probability of finding the liquid-like region is lower, i.e., there are few potential sites for the nucleation of the STZs. At higher strain rate, the lack of nucleation sites together with the shorter time for the activation result in a significant delay of the activation of STZs, which finally gives rise to a considerable stress overshoot. Therefore, the measured hardness at high strain rate is much larger than that obtained at low strain rate. This corresponds to the relatively large value of $m$ under a low peak-load. With increasing peak-load, the volume of material being sampled during indentation becomes larger, the probability of finding the potential STZ region is higher. In this case, the stress overshoot at higher strain rate will be weakened due to the abundant nucleation sites of the STZs. Then the difference of the hardness measured at different strain rate will be smaller. This corresponds to the decrease of $m$ with the increasing peak-load presented in Fig. 2a. Moreover, this also agrees well with the lower $m$ observed under macro-compression [32] than micro-pillar compression [33]. When the peak-load increases to larger than a critical value, the overall unstable plastic flow (the advent of a mature shear band) will occur immediately once the indenter presses into the metallic glass [20]. In such a scenario, the stress overshoot becomes further weak. This is consistent 
with the extremely small value of $m$ observed under $300 \mathrm{mN}$ and $20 \mathrm{~N}$ in the present work and the exceedingly weak dependence of yielding strength on the strain rate varying between $10^{-4} \mathrm{~s}^{-1}$ and $10^{-2} \mathrm{~s}^{-1}$ [26,32].

As mentioned above, for a fixed equivalent strain rate, a higher peakload corresponds to a larger indentation size within the same loading time. Then the probability of finding the potential STZ regions is higher. Consequently, the amount of activated STZs will be larger with increasing peak-load. Meanwhile, the larger amount of activated STZs will in turn lead to a higher net creation of free volume. During the indentation, the STZ and the free volume could prompt each other with a positive feedback [8]. As the activation of STZs and the creation of free volume always lead to a strain softening effect on the indentation hardness $[10,19]$, the combination of more activated STZs and higher free volume creation gives rise to a larger strain softening. This corresponds to the parabolic reduction of the indentation hardness with the increasing peak-load (or indentation depth) presented in Fig. 2b. When the peak-load increases to larger than a critical value, the overall plastic yielding will take place immediately. Further increasing of peak-load will only contribute to larger plastic deformation but not affect the yielding point, i.e., the hardness will level off in spite of the peak-load. This is analogous to the disappearance of ISE when the spherical indenter radius increased to larger than a critical value [20]. It is interesting to find that the increase of peak-load not only gives rise to the ISE and its disappearance but also results in an increase of the average length of pop-ins (Fig. 1d). As presented in Fig. 1a, the loading curves under different peak-load (smaller than $300 \mathrm{mN}$ ) can be treated as different stages of the same curve $(300 \mathrm{mN})$ because the loading segment under small peak-load perfectly coincides with that under large peakload. Then the variation of average pop-in length with the peak-load can be understood as the dependence of pop-in size on the indentation depth. As pointed out by Yang et al. [22], the displacement increment at a pop-in event $(\Delta h)$ is proportional to the indentation depth $(h)$ and can be written as: $\Delta h=\left(24.5 \Delta H / 2 K_{m}\right) h$, where $\Delta H$ is the hardness reduction at each pop-in event that is indeed approximately a constant throughout the entire indentation process. $K_{m}$ is a material constant. Accordingly, the pop-in size is expected to increase linearly with the indentation depth. In other words, the upper portion of the loading segment has a larger pop-in size. Therefore, the average pop-in length is larger under a higher peak-load. This is consistent with the increase of average pop-in length with the increasing peak-load observed in Fig. 1d.

In summary, based on a series of nanoindentations at different peakloads and strain rates, a positive dependence of hardness on the strain rate is found. Furthermore, the disappearance of both indentation size effect and strain rate influence on the hardness are also observed when the peak-load increases to larger than a critical value. The underlying mechanism is well understood in the framework of the activation and the accumulation of free volume via shear transformation zones. The present work is helpful to understand the dependence of hardness on the testing conditions (strain rate and peak-load) under nanoindentation and therefore provide a practical way to tailor the hardness of metallic glass.
Supplementary data to this article can be found online at http://dx. doi.org/10.1016/j.scriptamat.2017.05.046.

\section{Acknowledgements}

The financial support from the National Natural Science Foundation of China (NSFC) under Grant nos. 51171138, 11522221, 11372315 and 51321003 are gratefully acknowledged. This work was also supported by the foundation of National Ministry and Commission (granted no. $613262)$

\section{References}

[1] T.C. Hufnagel, C.A. Schuh, M.L. Falk, Acta Mater. 109 (2016) 375-393.

[2] A. Inoue, A. Takeuchi, Acta Mater. 59 (6) (2011) 2243-2267.

[3] Y. Gao, H. Bei, Prog. Mater. Sci. 82 (2016) 118-150.

4] L. Wang, H. Bei, Y.F. Gao, Z.P. Lu, T.G. Nieh, Acta Mater. 59 (7) (2011) 2858-2864.

[5] D.V. Louzguine-Luzgin, V.Y. Zadorozhnyy, S.V. Ketov, Z. Wang, A.A. Tsarkov, A.L. Greer, Acta Mater. 129 (2017) 343-351.

[6] A. Bhattacharyya, G. Singh, K.E. Prasad, R. Narasimhan, U. Ramamurty, Mater. Sci. Eng. A 625 (2015) 245-251.

[7] P. De Hey, J. Sietsma, A. Van Den Beukel, Acta Mater. 46 (16) (1998) 5873-5882.

[8] M.Q. Jiang, G. Wilde, L.H. Dai, Mech. Mater. 81 (2015) 72-83.

[9] A. Lemaître, Phys. Rev. Lett. 89 (19) (2002) 195503.

[10] N. Van Steenberge, J. Sort, A. Concustell, J. Das, S. Scudino, S. Suriñach, J. Eckert, M.D. Baró, Scr. Mater. 56 (7) (2007) 605-608.

[11] H.B. Bei, S.J. Xie, E.P. George, Phys. Rev. Lett. 96 (10) (2006) 105503.

[12] F. Xue, F. Wang, P. Huang, T.J. Lu, K.W. Xu, Mater. Sci. Eng. A 655 (2016) 373-378

[13] D. Pan, A. Inoue, T. Sakurai, M.W. Chen, Proc. Natl. Acad. Sci. U. S. A. 105 (39) (2008) 14769-14772.

[14] J. Sort, J. Fornell, W. Li, S. Surinach, M. Baro, J. Mater. Res. 24 (03) (2009) 918-925.

[15] T. Burgess, K.J. Laws, M. Ferry, Acta Mater. 56 (17) (2008) 4829-4835.

[16] L. Cheng, Z.M. Jiao, S.G. Ma, J.W. Qiao, Z.H. Wang, J. Appl. Phys. 115 (8) (2014), 084907

[17] Y. Ma, J.H. Ye, G.J. Peng, D.H. Wen, T.H. Zhang, Mater. Sci. Eng. A 627 (2015) $153-160$.

[18] Y. Ma, G.J. Peng, W.F. Jiang, H. Chen, T.H. Zhang, J. Non-Cryst. Solids 442 (2016) 67-72.

[19] J.-i. Jang, B.-G. Yoo, Y.-J. Kim, J.-H. Oh, I.-C. Choi, H. Bei, Scr. Mater. 64 (8) (2011) 753-756.

[20] S. Wang, Y.F. Ye, B.A. Sun, C.T. Liu, S.Q. Shi, Y. Yang, J. Mehc, Phys. Solids 77 (2015) 70-85.

[21] W.C. Oliver, G.M. Pharr, J. Mater. Res. 7 (06) (1992) 1564-1583.

[22] B. Yang, T.G. Nieh, Acta Mater. 55 (1) (2007) 295-300.

[23] J.C. Ye, J. Lu, C.T. Liu, Q. Wang, Y. Yang, Nat. Mater. 9 (8) (2010) 619-623.

[24] Z. Lu, X.N. Yang, B.A. Sun, Y.Z. Li, K. Chen, W.H. Wang, H.Y. Bai, Scr. Mater. 130 (2017) 229-233.

[25] C. Wang, Q.P. Cao, X.D. Wang, D.X. Zhang, U. Ramamurty, R.L. Narayan, J. Jiang, Adv. Mater. 29 (5) (2017) 1605537-1605542

[26] M.C. Li, M.Q. Jiang, S. Yang, F. Jiang, L. He, J. Sun, Mater. Sci. Eng. A 680 (2017) 21-26.

[27] Z.Y. Liu, Y. Yang, C.T. Liu, Acta Mater. 61 (16) (2013) 5928-5936.

[28] M.C. Li, M.Q. Jiang, G. Ding, Z.H. Peng, F. Jiang, L. He, J. Sun, J. Non-Cryst. Solids 468 (2017) 52-57.

[29] M.Q. Jiang, G. Wilde, L.H. Dai, Scr. Mater. 127 (2017) 54-57.

[30] Y. Kawamura, T. Shibata, A. Inoue, T. Masumoto, Appl. Phys. Lett. 71 (6) (1997) 779-781.

[31] N. Koumakis, M. Laurati, S.U. Egelhaaf, J.F. Brady, G. Petekidis, Phys. Rev. Lett. 108 (9) (2012) 098303-098307.

[32] Y.W. Wang, X.L. Bian, S.W. Wu, I. Hussain, Y.D. Jia, J. Yi, G. Wang, Sci. Rep. 6 (2016) 27747.

[33] D. Tönnies, R. Maaß, C.A. Volkert, Adv. Mater. 26 (32) (2014) 5715-5721. 